TUW-93-19

\title{
On the definiteness of the conformal anomaly in
}

\section{nonconformal gauges}

\author{
W. Mödritsch \\ Institut für Theoretische Physik, TU-Wien \\ 1040 Wien, Wiedner Hauptstraße 8-10/136, Austria \\ FAX: (+43-1) 567760, e-mail: wmoedrit@ecxph.tuwien.ac.at
}

\begin{abstract}
The critical dimension of the bosonic string in the harmonic and the deDonder gauge may be calculated from the time ordered product of two energy momentum tensors. We show that recently found ambiguities within that method in nonconformal gauges can be resolved by a treatment respecting background covariance.
\end{abstract}

PACS: 11.17 
The well-known central feature of the bosonic string is that it can only consistenly be formulated in 26 dimensions. While this result is well established in the usual conformal gauge, there are only a few calculations verifying this result in nonconformal gauges [1, 2, 3, 河. However, it was claimed recently [5] that due to ambiguities in the energy momentum tensor (e-m tensor) in those gauges, it may happen that the coefficient of the conformal anomaly cannot be computed in a unique way. This would be a serious contradiction to existing theorems which prove the gauge-independence of anomalies, even including anomalies of global symmetries in general gauge theories [6], or to a theorem which states that the anomalous vertex is the time ordered product of two e-m tensors [4]. According to those theorems, anomalies in different gauges can only differ by terms which may be absorbed as counter terms to the action.

It is the purpose of this note to show that by using a uniquely defined background covariant e-m tensor the usual result is obtained, also with the method of 5 . Two different (nonconformal) gauges are studied for this purpose.

To the free bosonic string action $\left(g=-\operatorname{det} g_{\alpha \beta}\right)$

$$
S_{x}=\frac{1}{2} \int d^{2} x \sqrt{g} g^{\alpha \beta} \partial_{\alpha} X^{M} \partial_{\beta} X_{M}
$$

we add a Faddeev-Popov and gauge fixing term in order that diffeomorphism and also Weyl rescalings are fixed completely:

$$
S_{\phi \pi}+S_{g f}=i \int d^{2} x s\left[\bar{c}_{\alpha} F^{\alpha}(g, \hat{g})+\bar{\tau} F(g, \hat{g})\right]
$$


We have introduced the nilpotent BRST operator $s$ :

$$
\begin{aligned}
& s g^{\alpha \beta}=-\partial_{\lambda} c^{\alpha} g^{\lambda \beta}-\partial_{\lambda} c^{\beta} g^{\lambda \alpha}+c^{\lambda} \partial_{\lambda} g^{\alpha \beta}-\tau g^{\alpha \beta} \\
& s c^{\alpha}=c^{\lambda} \partial_{\lambda} c^{\alpha}, \quad s \tau=c^{\lambda} \partial_{\lambda} \tau \\
& s X^{M}=c^{\lambda} \partial_{\lambda} X^{M} \\
& s \bar{c}_{\alpha}=i B_{\alpha}, \quad s \bar{\tau}=i B
\end{aligned}
$$

The harmonic gauge is defined by the gauge fixing conditions (cf. [4, 2, 3])

$$
\begin{aligned}
F^{\alpha}(g, \hat{g}) & =\hat{\nabla}_{\beta} \sqrt{g} g^{\alpha \beta} \\
F(g, \hat{g}) & =\sqrt{\hat{g}} \hat{g}^{\alpha \beta} h_{\alpha \beta},
\end{aligned}
$$

where we have choosen the gauge fixing metric to be equal to the classical part of $g_{\alpha \beta}$, which need not be the case in general. This means that

$$
h_{\alpha \beta}=g_{\alpha \beta}-\hat{g}_{\alpha \beta}
$$

is our quantum field. As shown in [3] it is possible to linearize the action with respect to $h$ without changing the physics because only one-loop graphs contribute since $h$ does not propagate - except by changing into $B$. Furthermore eq. (5) leads to an algebraic equation for $h$ and can integrated out immediately. This means that $h$ has to be considered as traceless with respect to $\hat{g}$ in the following. For the calculation of the time ordered product of two e-m tensors to one loop order we only need the part from the action which is bilinear in the fields. Then the diffeomorphism ghost and gauge fixing part (2) becomes

$$
S_{\phi \pi}+S_{g f}=\int d^{2} x \sqrt{\hat{g}}\left[i \bar{c}_{\alpha}\left(\hat{\nabla}_{\gamma} \hat{\nabla}^{\gamma}-\frac{1}{2} \hat{R}\right) c^{\alpha}+B_{\alpha} \hat{\nabla}_{\beta} h^{\alpha \beta}\right]
$$


The e-m tensor

$$
T_{\alpha \beta}=\frac{2}{\sqrt{\hat{g}}} \frac{\delta S}{\delta \hat{g}^{\alpha \beta}}
$$

is by definition conserved with respect to the background covariant derivative. (8) will be discussed further below. With eqs. (7) and (8) the ghost and $B-h$ parts of the e-m tensor are obtained in an unambiguous way.

$$
\begin{gathered}
T_{\alpha \beta}^{\bar{c} c}=2 i\left[\hat{\nabla}_{(\alpha} \bar{c}^{\gamma} \hat{\nabla}_{\beta)} c_{\gamma}-\hat{\nabla}^{\gamma} \bar{c}_{(\alpha} \hat{\nabla}_{\gamma} c_{\beta)}+\hat{\nabla}_{\gamma}\left(\bar{c}^{\gamma} \hat{\nabla}_{(\alpha} c_{\beta)}+\hat{\nabla}_{(\alpha} \bar{c}_{\beta)} c^{\gamma}\right)-\right. \\
-\frac{g_{\alpha \beta}}{2}\left(\hat{\nabla}^{\gamma} \bar{c}^{\sigma} \hat{\nabla}_{\gamma} c_{\sigma}+\hat{\nabla}_{\gamma} \hat{\nabla}_{\sigma}\left(\bar{c}^{\sigma} c^{\gamma}\right)+\bar{c}^{\sigma} R_{\sigma \gamma} c^{\gamma}\right)- \\
\left.-\bar{c}_{(\alpha}\left(\hat{\nabla}_{\gamma} \hat{\nabla}^{\gamma}-\frac{1}{2} \hat{R}\right) c_{\beta)}\right] \\
T_{\alpha \beta}^{B h}=\quad-2\left(\hat{\nabla}_{(\alpha} B^{\gamma}\right) h_{\beta) \gamma}-B^{\gamma} \hat{\nabla}_{\gamma} h_{\alpha \beta}+\hat{g}_{\alpha \beta} \hat{g}^{\mu \nu}\left(\hat{\nabla}_{\mu} B^{\gamma} h_{\nu \gamma}\right)+2 B_{(\alpha} \hat{\nabla}^{\sigma} h_{\sigma \beta)}
\end{gathered}
$$

The last terms of these e-m tensors are essentially equations of motion and could have been avoided, if we had decided to take $\bar{c}^{\alpha}$ and $B^{\alpha}$ as $\hat{g}$ independent fields, instead of $\bar{c}_{\alpha}$ and $B_{\alpha}$. They do not contribute to the anomaly.

The computation of the conformal anomaly starts from the flat limit of $\hat{g}$ in (9) and (10). Before stating the result of this calculation, we turn to the method used in [5]. There $\hat{g}$ was choosen to be flat already at the level of the action. Ambiguities may arise in this case because by covariantizing ordinary derivatives, ordering problems appear. Consider the term with $\hat{R}$ in $(\overline{0})$. It obviously vanishes in the flat limit, but its contribution to the e-m tensor is readily calculated as 


$$
\begin{aligned}
\lim _{\hat{g} \rightarrow \eta} T_{\alpha \beta}^{R}:= & -\lim _{\hat{g} \rightarrow \eta} i \frac{2}{\sqrt{\hat{g}}} \frac{\delta}{\delta \hat{g}^{\alpha \beta}} \int d^{2} x \sqrt{\hat{g}} \bar{c}^{\mu} \hat{R}_{\mu \nu} c^{\nu}= \\
= & -\lim _{\hat{g} \rightarrow \eta} i\left[\hat{\nabla}_{\rho} \hat{\nabla}_{(\alpha}\left(\bar{c}_{\beta)} c^{\rho}\right)+\hat{\nabla}_{\rho} \hat{\nabla}_{(\alpha}\left(\bar{c}^{\rho} c_{\beta)}\right)-\hat{\nabla}_{\rho} \hat{\nabla}^{\rho}\left(\bar{c}_{(\alpha} c^{\beta)}\right)-\right. \\
& \quad-\hat{g}_{\alpha \beta}\left(\hat{\nabla}_{\mu} \hat{\nabla}_{\nu}\left(\bar{c}^{\mu} c^{\nu}\right)+\bar{c}^{\mu} \hat{R}_{\mu \nu} c^{\nu}\right)+\hat{R}_{\left.{ }_{\alpha} c_{\beta)}\right]=} \\
= & -i\left\{\partial_{\sigma}\left[\partial_{(\alpha}\left(\bar{c}^{\sigma} c_{\beta)}+\bar{c}_{\beta)} c^{\sigma}\right)-\partial^{\sigma}\left(\bar{c}_{(\alpha} c_{\beta}\right)\right]-\eta_{\alpha \beta} \partial_{\sigma} \partial_{\rho}\left(\bar{c}^{\sigma} c^{\rho}\right)\right\}
\end{aligned}
$$

This turns out to exactly coincide with the ambiguous term of ref [5]. Thus, in a covariant approach, this term must not be traced back to a total derivative in the action, but to the curvature term above. It should be stressed that the factor in front of this term is completely determined by the gauge-choice which led to (7) so that no ambiguity remains if the limit $\hat{g} \rightarrow \eta$ is taken after computing (9) and (10) and not before.

We also note that (11) is symmetric and conserved without the equations of motion in the flat limit

$$
\partial^{\alpha} \lim _{\hat{g} \rightarrow \eta} T_{\alpha \beta}^{R}=0
$$

This situation appears to be the analogue of the well known fact that in the conformal gauge one can add to the flat e-m tensor the quantity

$$
t_{++}=\partial_{+}\left(c^{+} b_{++}\right)
$$

which is conserved by virtue of the equations of motion. The coefficient of this quantity is also only fixed by performing the calculation on the curved world sheet. Furthermore, Eq. (13) can be derived also from a curvature term if the ghost action is formulated in 
the bosonized language [7].

In our fully background covariant approach we now calculate the anomaly from the flat limit of the e-m tensor (9) $+(10)$, including the usual part from the $X$ fields by considering the nonlocal pieces of the time ordered product of two e-m tensors. The result is

$$
\left\langle T T_{\alpha \beta}(x) T_{\gamma \delta}(y)\right\rangle=\frac{i c}{12 \pi} \int d^{2} k e^{i k(x-y)} \frac{k_{\alpha} k_{\beta} k_{\gamma} k_{\delta}}{k^{2}}+\text { local terms }
$$

with the constant $c$ given in tab. 1 where $X, c \bar{c}$, and $B h$ denote the respective contribution of the loops with boson, F.P. ghost and with $B-h$ propagators. The fact that the time ordered product of two e-m tensors as defined by eq. (8) is related to the anomalous vertex can also be understood from another point of view. If one recognizes the effective action as the underlying theoretical concept, one can show [1] that the second derivative of the effective action with respect to the metric $\hat{g}$ (the classical part of $g$ ) is the anomalous vertex. Since the nonlocal part of that quantity is equal to the nonlocal part of eq. (14), if the e-m tensor is defined as in eq. (8), this argument shows that we have calculated the usual conformal anomaly.

As a further illustration of the anomaly calculation in non-conformal gauges in the operator formalism we choose the deDonder gauge:

$$
\begin{aligned}
F^{\alpha}(g, \hat{g}) & =\partial_{\beta}\left(\hat{g}^{\alpha \beta}-g^{\alpha \beta}\right)=\partial_{\beta} h^{\alpha \beta} \\
F(g, \hat{g}) & =\hat{g}^{\alpha \beta} h_{\alpha \beta}
\end{aligned}
$$

The linearized action of the ghost and auxiliary fields (without interaction terms and with 
the Weyl ghosts integrated out) reads:

$$
S_{\phi \pi}+S_{g f}=\int d^{2} x\left[i \bar{c}_{\beta} \partial_{\alpha}\left(2 \hat{\nabla}^{(\alpha} c^{\beta)}-\hat{g}^{\alpha \beta} \hat{\nabla}_{\rho} c^{\rho}\right)-B_{\beta} \partial_{\alpha} h^{\alpha \beta}\right]
$$

It should be mentioned that the flat limit of this part of the action is the same as that of eq. (7). However, the two e-m tensors differ. In the approach of refs. [2, [3] and [4] this is reflected by the fact that the vertices of the background field depend on the gauge.

The e-m tensor derived with (8) from the action (17) reads

$$
\begin{aligned}
T_{\alpha \beta}^{\bar{c} c}= & \frac{2}{\sqrt{\hat{g}}}\left[\partial_{(\alpha} \bar{c}_{\mu} \partial_{\beta)} c^{\mu}+\partial_{\mu} \bar{c}_{\alpha} \partial_{\beta)} c^{\mu}+\partial_{\mu} \partial_{(\alpha} \bar{c}_{\beta)} c^{\mu}+\frac{1}{2} \partial_{(\alpha} \bar{c}_{\beta)} \hat{g}_{\mu \nu} \partial_{\lambda} \hat{g}^{\mu \nu} c^{\lambda}-\right. \\
& \left.-\frac{1}{2} \hat{g}^{\mu \nu} \partial_{\mu} \bar{c}_{\nu} \hat{g}_{\rho(\alpha} \hat{g}_{\beta) \sigma} \partial_{\lambda} \hat{g}^{\rho \sigma} c^{\lambda}-\frac{1}{2} \partial_{\lambda}\left(\hat{g}_{\alpha \beta} \hat{g}^{\mu \nu} \partial_{\mu} \bar{c}^{\nu} c^{\lambda}\right)\right] \\
T_{\alpha \beta}^{B h}= & \frac{1}{\sqrt{\hat{g}}} \hat{g}^{\mu \nu} \partial_{\mu} B_{\nu} h_{\alpha \beta}
\end{aligned}
$$

For the $B$ - $h$ part one has to take into account the tracelessnes of $h$. The contributions to the anomaly coefficient for both gauges are given in tab. 1 with $X, \bar{c} c$ and $B h$ denoting the respective loop contributions.

\begin{tabular}{c|c|c|c|c} 
gauge & $X$ & $\bar{c} c$ & $B h$ & net result \\
\hline harmonic & $D$ & -52 & 26 & $D-26$ \\
deDonder & $D$ & -28 & 2 & $D-26$
\end{tabular}

Tab1.: Anomly coefficient $c$ (eq. (14))

These results are in agreement with those of ref. [3] obtained by calculating the effective action and the result for the harmonic gauge is in agreement with ref. [1], too. The general theorems on the gauge independence of anomalies [6] are thus seen to hold true. 


\section{Acknowledgements}

The author has profited from discussions with H. Balasin, W.Kummer, H. Nachbagauer, A. Rebhan and S. Sorella. He thanks W.Kummer and A. Rebhan for a critical reading of the manuscript and useful suggestions regarding the latter.

\section{References}

[1] D.W. Düsedau, Phys.Lett. B188, 51 (1987);

D.Z. Freedman, J.I. Latorre and K. Pilch, Nucl.Phys. B306, 77 (1988).

[2] A. Rebhan and U. Kraemmer, Phys.Lett. B196, 477 (1987).

[3] U. Kraemmer and A. Rebhan, Nucl.Phys. B315, 717 (1989)

[4] L. Baulieu and A. Bilal, Phys.Lett. B192, 339 (1987).

[5] M. Abe and N. Nakanishi, Mod.Phys.Lett. A7, 1799 (1992).

[6] O. Piguet and K. Sibold, Nucl.Phys. B248,301,336 (1984);

W. Kummer, Lecture at XXIX Int. Universitätswoche für Kernphysik, 1990 in Schladming, Austria; Phys.Lett. B231, 231 (1989);

O. Piguet, D.Schwarz and M. Schweda, Phys.Lett. B245, 393 (1990).

[7] M.B. Green, J.H. Schwarz, E. Witten "Superstring Theory" Vol.1, Combridge Univ. Press, 1987. 